\title{
On the Dimension Group of Classical Physics
}

\author{
Chester H. Page \\ Institute for Basic Standards, National Bureau of Standards, Washington, D.C. 20234 \\ (May 15, 1975)
}

\begin{abstract}
The basic principles of the group properties of physical quantities are reviewed. The problems associated with the dimensions of angle and logarithm are solved by using functional equations, instead of analytic expressions, for defining functions of nonnumerical quantities. It is concluded that the neper and radian are related by $\mathrm{Np}=-\mathrm{j}$ rad, so that when the radian is considered as a base unit, the neper becomes a derived unit, and is the SI unit for logarithmic quantities.
\end{abstract}

Key words: Angle; dimension; logarithm; neper; radian.

\section{Introduction}

Theoretical physics and engineering are based on mathematical models of the phenomena of nature, i.e., the relations among measurable physical entities are expressed by mathematical equations. Scientists originally considered these equations to be relations among numbers, with the numbers representing the measures of quantities expressed in terms of agreed-upon units. Maxwell was one of the first to realize that this interpretation of equations in science is sterile compared to the quantity calculus concept. In the modern quantity equation viewpoint, the letter symbols in an equation represent mathematical elements, called quantities, which are in a one-toone correspondence with the measurable entities of the laboratory.

In measurement, the physical entity corresponding to the mathematical element (quantity) $q$ is measured by its ratio to a standard entity sample called a measurement unit. The mathematical counterpart of this measurement unit is the symbolic unit, $[q]$. The result of a measurement is thus modeled by the relation

$$
q=\{q\}[q]
$$

expressing the values of the quantity as the product of a positive real number, $\{q\}$ (the measure or numerical value of the quantity), and the chosen unit. Since the laws of physics are observed as proportionalities among measures, these laws are modeled by multiplicative relations.

If, experimentally, the measure of a quantity $q$ is proportional to the product of the measures of the quantities $p$ and $s$, the experimenter's measure equation

$$
\{q\}=k\{p\}\{s\}
$$

can be generalized to the theoretical quantity equation

$$
q=n p s
$$

where $q, p, s$ are mathematical elements in the dimension spaces $D(q), D(p), D(s)$, and $n$ is a number. $D(q)$ is commonly called "the dimension of $q$ ". By taking the dimension space of number, $D(n)$, as the identity element, the set of dimensions becomes an Abelian group. The "product"

AMS Subject Classification: 2000. 
$p s$ is not yet defined. However, a postulate on the completeness of a physical law (see reference $\left.[1]^{1}\right)$ implies that the product operation has the following property:

"The product of any point in $D(p)$ and any point in $D(s)$ is a point in $D(q)$, and conversely, any point in $D(q)$ can be expressed as the product of some point in $D(p)$ and some point in $D(s)$."

It follows that

$$
D(p s)=D(p) D(s)
$$

The quantity equation can be written

$$
\{q\}[q]=n\{p\}\{s\}[p][s]
$$

If the units of $q, p$, and $s$ are chosen to make $n=k$, then

$$
[q]=[p][s]
$$

and the system of units is said to be coherent (strictly speaking, coherent with the system of quantities and equations used to represent the theory).

The number of independent dimensions is a matter of taste and convenience. In the International System of Units, those "units which by convention are regarded as dimensionally independent" [2] are called "base units."

\section{Supplementary Units}

The dimension space of "angle" is frequently taken as identical to the dimension space of numbers; the quantity, angle, is then numeric, and its unit, radian, can be interpreted as a special name for the number 1. On the other hand, it is often convenient to distinguish "work" and "torque" as being entities of different kinds, by assigning them to different dimension spaces. In this case, angles are not numbers, and the radian is a base unit. The General Conference on Weights and Measures has very properly declined to make a dogmatic decision on this question of taste and convenience, so has classified the radian (and steradian) as supplementary units in the SI.

The International Organization for Standardization (ISO), although it no longer recognizes the use of radian as a base unit, formerly gave two definitions of "angle." One of these definitions was based on the concept of "analytical angle," defined as the ratio of the arc cut out on a circle to the radius of that circle. This concept makes "angle" a pure number. The second definition was based on the concept of "geometrical angle," which expresses the degree of divergence between two intersecting straight lines. In this concept, which is usually used in technology, "angle" possesses its own nature and dimension, and the radian is a base unit.

The engineering practice of making a dimensional distinction between "number" and "angle" is related to the existence, and use, of a multiplicity of units. An angle cannot be completely described by a number alone; the unit (radian, degree, grade, etc.) must also be stated. It is therefore meaningless to say, for example, that a certain angle is greater than 2 .

\section{Logarithmic Quantities}

The nature of the units and dimensions of "logarithmic quantities" such as attenuation and level raises problems similar to those raised by "angle." The attenuation of a "signal" (communication signal, light beam, electric power, etc.) can be measured by comparison with the attenuation associated with a standard reference situation.

' Figures in brackets indicate the literature references at the end of the paper. 
A l-bel standard for attenuation in a transmission path makes the output (transmitted) power $1 / 10$ of the input (incident) power and makes the output voltage and current $\sqrt{1 / 10}$ of the input voltage and current. A 1-neper standard makes the output power $1 / \mathrm{e}^{2}$ of the input power, and the output voltage and current $1 / \mathrm{e}$ of the input voltage and current. Thus a given attenuation can be expressed as, say, 1 bel (commonly given as 10 decibels) or as 1.151 nepers. The given quantity, attenuation, is expressed as the product of a numerical value and a unit, and the prevalence of two different units makes such a statement as "The attenuation was 3 " an incomplete statement. The quantity, attenuation, is therefore not a pure number, but is an element in some other dimension space.

Since attenuation.can be expressed as the logarithm of the ratio of two values of a quantity, it is the logarithm of a number. This creates the paradoxical situation wherein a nonnumerical dimension is assigned to a function of a pure number.

The major object of this paper is to resolve this paradox, i.e., to explore the existence of possible pairings of numeric and nonnumeric functions of numeric and nonnumeric variables.

\section{Structure of the Group of Quantities}

A set of coherent units forms a group which is isomorphic to the group of dimensions. Since the primitive quantities can be expressed as products of units by positive real numbers, they constitute the direct product group $\mathscr{P} \times \mathscr{G}$, where $\mathscr{P}$ is the group of positive real numbers, and $\mathscr{G}$ the Abelian multiplicative group of units. For mathematical treatment of quantities, it is necessary to choose reference directions or polarities, creating negative measures. The quantity group becomes $\mathscr{R} \times \mathscr{G}$, where $\mathscr{R}$ is the field of real numbers.

The group $\mathscr{R} \times \mathscr{G}$ has the following properties:

If $a u$ denotes the element in $\mathscr{R} \times \mathscr{G}$ determined by the element $a$ of $\mathscr{R}$ and the element $u$ of $\mathscr{G}$, then

$$
(a+b) u=a u+b u
$$

and

$$
(a u)(b v)=(a b) u v
$$

hold for any $a, b$ in $\mathscr{R}$ and any $u, v$ in $\mathscr{G}$.

Relations like

$$
a u+b v=c u+d v
$$

can always be decomposed into independent relations:

$$
\begin{aligned}
& a u=c u \Rightarrow a=c \\
& b v=d v \Rightarrow b=d
\end{aligned}
$$

and the equations of physics can be written in dimensionally homogeneous form.

In physics and engineering, it is frequently convenient, for mathematical manipulation, to use complex numbers. Basically, this is an artifice to allow the simultaneous treatment of pairs of complementary quantities. The familiar elementary example is the simultaneous treatment of sine and cosine components of an oscillatory solution of a differential equation. This is a special case of a general artifice using hypercomplex quantities to simultaneously manipulate related quantities having different dimensions [3]. The usual occurrence of this device is in the form

$$
a u+\mathrm{j} b v
$$

where $a$ and $b$ are real numbers, and the unit imaginary, $\mathrm{j}$, serves as a redundant but useful "flag."

We first examine the implications of allowing angle to be nonnumeric. If we define $\cos \theta$ and $\sin$ $\theta$, as quantities, by 


$$
\begin{array}{r}
\cos \theta=\sum_{n=0}^{\infty} \frac{(-1)^{n} \theta^{2 n}}{2 n !} \\
\sin \theta=\sum_{n=0}^{\infty} \frac{(-1)^{n} \theta^{2 n+1}}{(2 n+1) !}
\end{array}
$$

then each term of the expansion on the right must have the same dimension, and this must be the dimension of the quantity on the left. This "dimensional homogeneity" requires

$$
\begin{gathered}
D^{2}(\theta)=D(\theta) D(\theta)=D(1) \\
D(\cos \theta)=D(1) \\
D(\sin \theta)=D(\theta)
\end{gathered}
$$

If we interpret eq (9) to be only numerical equations with $\theta$ representing the measure of the angle relative to the radian as a unit, no such conclusion can be drawn. In that case, we must consider some other definition of angle as a quantity, which is compatible with (9) as a relation among numerical values.

Now $\cos \theta$ and $\sin \theta$ can also be defined as appropriate solutions of the differential relations

$$
\begin{gathered}
\mathrm{d} \sin \theta=\cos \theta \mathrm{d} \theta \\
\mathrm{d} \cos \theta=-\sin \theta \mathrm{d} \theta
\end{gathered}
$$

In this case, dimensional homogeneity requires

$$
\begin{aligned}
& D(\sin \theta)=D(\cos \theta) D(\theta) \\
& D(\cos \theta)=D(\sin \theta) D(\theta)
\end{aligned}
$$

which again implies

$$
D^{2}(\theta)=D(1)
$$

Note, however, that if $D(\theta) \neq D(1)$, then $D(\sin \theta) \neq D(\cos \theta)$, and the dimensions of various functions of $\theta$ are not known a priori; in particular, functions of $\theta$ are not necessarily numerical.

\section{Classes of Functions in $\mathscr{R} \times \mathscr{G}$}

Denote the unity element of $\mathscr{G}$ by 1 . Since 1 is idempotent, a function $f(x)$ defined on $\mathscr{R}$ can also be considered as defined on $\mathscr{R} \times 1$ :

$$
f(x) \epsilon \mathscr{R} \times 1, \quad x \epsilon \mathscr{R} \times 1
$$

Generalization to $f(x)$ with $f \epsilon \mathscr{R} \times \mathscr{G}$ and $x \epsilon \mathscr{R} \times \mathscr{G}$ develops the additional classes of functions:

$$
\begin{array}{cc}
F(x) \epsilon \mathscr{R} \times \mathscr{G}, & x \epsilon \mathscr{R} \times 1 \\
f(X) \epsilon \mathscr{R} \times 1, & X \epsilon \mathscr{R} \times \mathscr{G} \\
F(X) \epsilon \mathscr{R} \times \mathscr{G}, & X \epsilon \mathscr{R} \times \mathscr{G}
\end{array}
$$


The use of lower-case letters for elements of $\mathscr{R}($ or $\mathscr{R} \times 1)$ and capital letters for general elements of $\mathscr{B} \times \mathscr{G}$ will simplify the presentation, and avoid the need for repeated statements about domains and ranges.

Since multiplication is defined in $\mathscr{R} \times \mathscr{G}$, multiplication of non-numerical quantities generates trivial examples of Class D. Generalization of other functions will be based on implicit definitions provided by functional equations, in particular, by addition theorems and multiplication theorems possessed on $\mathscr{R}$.

For classes $\mathrm{C}$ and $\mathrm{D}$, multiplication theorems are inappropriate; addition theorems are required, with the terms of the argument containing the same factor from $\mathscr{G}$. For class B, a multiplication theorem is required to separate this class from $\mathrm{D}$ by restricting the domain.

The lack of addition on $\mathscr{G}$ necessitates dimensional homogeneity in equations; the general multiplication and addition theorems for classes (B), (C), and (D) are:

$$
\begin{gathered}
F(x y)=g(x, y) F(y)+g(y, x) F(x) \\
f(X+Y)=h\{f(X), f(Y)\}+h\{f(Y), f(X)\} \\
F(X+Y)=g(X, Y) F(Y)+g(Y, X) F(X)
\end{gathered}
$$

where

$$
\begin{aligned}
& X=\{X\} U \\
& Y=\{Y\} U
\end{aligned}
$$

and $U \boldsymbol{\epsilon} \mathscr{G}$.

Although $F$ is a mapping of $\mathscr{R}$ onto $\mathscr{R} \times \mathscr{G}$, it is implicitly defined by a functional equation $E(F)$ in its own image space. The inverse mapping is similarly defined by an equation $e(f)$ in its image space. The mappings thus give

$$
\begin{array}{cl}
F: \mathscr{R} \rightarrow \mathscr{R} \times \mathscr{G}, & e(f) \rightarrow E^{\prime}(F) \\
f: \mathscr{R} \times \mathscr{G} \rightarrow \mathscr{R}, & E(F) \rightarrow e^{\prime}(f) .
\end{array}
$$

The relations so far are completely general, permitting an infinity of functions satisfying pairs of multiplication/addition theorems. If we wish to select a set of functions having special properties, the simplest and most interesting set comprises those functions having unique functional theorems, i.e., $E^{\prime}(F) \equiv E(F), e^{\prime}(f) \equiv e(f)$ so that

Since

$$
\begin{aligned}
& F: e(f) \rightarrow E(F) \\
& f: E(F) \rightarrow e(f) .
\end{aligned}
$$

$$
F(f(X+Y)) \equiv X+Y=F(f(X))+F(f(Y))
$$

the inverse of (14) is

where

$$
F(x)+F(y)=F\{h(x, y)+h(y, x)\}
$$

$$
\begin{gathered}
\mathbf{x} \equiv f(X) \\
X \equiv F(x) .
\end{gathered}
$$

For (17) and (13) to have identical form, (14) and (13) must be 


$$
\begin{aligned}
& f(X+Y)=f(X) f(Y) \\
& F(x y)=F(x)+F(y)
\end{aligned}
$$

The $\mathscr{G}$-component of $F$ (the unit of $F$ ) can be factored out of (18b), making the equation appear to be a trivial combination of the solution of

$$
f(x y)=f(x)+f(y)
$$

and an arbitrary unit of $\mathscr{G}$. For real $x$, (19) has the well-known solution

$$
f(x)=\log _{a} x
$$

where the logarithm may be taken to any base.

Since $f(x)$ is the measure $\{F(x)\}$, its different numerical values associated with different logarithmic bases may be interpreted as different measures corresponding to different choices of the size of the unit of $F$, that is, to different choices of $a$ when $F(a)$ is the sample taken as the unit of $F$.

Since the quantity $F(x)$ is invariant to unit choice, we can write

$$
F(x)=\{F(x)\}_{a} F(a)=\{F(x)\}_{b} F(b)
$$

where $\{F\}_{a}$ denotes the measure of $F$ when $F(a)$ is the unit. In terms of $F(b)$ as the unit,

$$
F(a)=\{F(a)\}_{b} F(b)
$$

Combining (21) and (22) yields

$$
\frac{\{F(x)\}_{b}}{\{F(x)\}_{a}} \equiv\{F(a)\}_{b}
$$

as the relation between different measures of $F(x)$.

Now

$$
\frac{\log _{b} x}{\log _{a} x} \equiv \log _{b} a
$$

so that the solution $(20)$ can be written

$$
\{F(x)\}_{a}=\log _{a} x
$$

implying that $F(x)$ is a generalized nonnumerical logarithm:

$$
\begin{aligned}
F(x) & =\operatorname{LOG} x \\
& =\log _{a} x \text { LOG } a
\end{aligned}
$$

with LOG $a$ an element of $\mathscr{G}$. The engineering usages of neper $(\mathrm{Np})$ and bel as units are interpretable as usage of LOG e and LOG $\sqrt{10}$ as units, with the quantity, attenuation (gain, loss), defined by LOG $\sqrt{P_{1} / P_{2}}$, where $P_{1}$ and $P_{2}$ are the power at points 1 and 2 of a system:

$$
\text { LOG } \mathrm{e}=\mathrm{Np}
$$


With the introduction of the complex number artifice, (18b) has also the solution

$$
F_{2}(x)=\operatorname{ARG} x \stackrel{d}{=} \operatorname{ARCTAN} \frac{\operatorname{Im} x}{\operatorname{Re} x}
$$

For the inverse to be unique, the modulus of $x$ must be fixed, conveniently at unity. The "natural" unit of $F_{2}$ is given by

$$
\operatorname{ARG} \mathrm{e}^{\mathrm{j}}=\mathrm{rad}
$$

Equations (18) have, therefore, two primitive solutions:

(1) For $x, y \in \mathscr{R}$,

$$
F_{1}(x)=\operatorname{LOG} x
$$

mapping the multiplicative group $\mathscr{R}$ onto an additive group $\mathscr{L}$ of logarithms:

$$
\begin{aligned}
& F_{1}(\mathscr{R})=\mathscr{L} \\
& f_{1}(\mathscr{L})=\mathscr{R}
\end{aligned}
$$

(2) For $x, y \epsilon \mathscr{C}_{1}$ (the multiplicative group of complex numbers of unit modulus)

$$
\begin{gathered}
F_{2}(x)=\operatorname{ARG} x \stackrel{d}{=} \operatorname{ARCTAN} \frac{\operatorname{Im} x}{\operatorname{Re} x} \\
F_{2}\left(\mathscr{C}_{1}\right)=\Theta \\
f_{2}(\Theta)=\mathscr{C}_{1}
\end{gathered}
$$

where $\Theta$ is the additive group of angles.

These two primitive solutions can be combined into a general solution:

$$
\begin{gathered}
F(\mathscr{C})=F\left(\mathscr{R} \times \mathscr{C}_{1}\right)=F(\mathscr{R}) \cup F\left(\mathscr{C}_{1}\right)=F_{1}(\mathscr{R}) \cup F_{2}\left(\mathscr{C}_{1}\right)=\mathscr{L} \cup \Theta \\
f(\mathscr{L} \cup \Theta)=f(\mathscr{L}) \times f(\Theta)=f_{1}(\mathscr{L}) \times f_{2}(\Theta)=\mathscr{R} \times \mathscr{C}_{1}=\mathscr{C}
\end{gathered}
$$

where $\mathscr{C}$ is the complex number field. Thus $F$ maps the product of the two multiplicative groups $\mathscr{R}$ and $\mathscr{C}_{1}$ onto the union of the two additive groups $\mathscr{L}$ and $\Theta$.

Equation (18a) implies

$$
f(n X)=(f(X))^{n}
$$

for integral $n$.

Since $f(X) \epsilon \mathscr{C}$, the right hand side of (34) is defined for general complex numbers $z$ in place of $n$. Generalization of (34) yields

$$
f(z X)=(f(X))^{z}
$$

Now (27) and (29) imply, respectively, 


$$
\begin{aligned}
& f(\mathrm{~Np})=\mathrm{e} \\
& f(\mathrm{rad})=e^{\mathrm{j}}
\end{aligned}
$$

so that $(35)$ yields

$$
\begin{gathered}
f(\mathrm{jNp})=\mathrm{e}^{\mathrm{j}}=f(\mathrm{rad}) \\
f(-\mathrm{j} \mathrm{rad})=\mathrm{e}^{1}=f(\mathrm{~Np})
\end{gathered}
$$

or

$$
\mathrm{Np} \equiv-\mathrm{j} \text { rad }
$$

Thus when the radian is considered to be a base unit, the neper is dimensionally the same as a radian, if the imaginary $\mathrm{j}$ is considered dimensionally numeric. When radian is considered to be a special name for the number 1 , neper becomes a special name for $-\mathrm{j}$, not a special name for the number 1 as is sometimes stated.

Equation (38) also implies that

$$
\mathscr{L} \equiv \mathscr{R} \times[\mathrm{LOG}]=\mathrm{j} \mathscr{R} \times \Theta
$$

and since

$$
\Theta \equiv \mathscr{R} \times \Theta
$$

we have

$$
\mathscr{L} \cup \Theta=\mathscr{C} \times \theta
$$

so that

$$
\begin{aligned}
& F(\mathscr{C})=\mathscr{C} \times \Theta \\
& f(\mathscr{C} \times \boldsymbol{\theta})=\mathscr{C} .
\end{aligned}
$$

Thus $F$ is a mapping of complex numbers onto complex angles.

In particular, measuring in radians and nepers,

$$
\begin{gathered}
f(\theta)=f(\{\theta\} \operatorname{rad})=(f(\operatorname{rad}))^{\{\theta\}}=\mathrm{e}^{\mathrm{j}\{\theta\}}=\mathrm{e}^{\mathrm{j} \theta / \mathrm{rad}} \\
f(L)=f(\{L\} \mathrm{Np})=\mathrm{e}^{\{L\}}=\mathrm{e}^{L / \mathrm{Np}}
\end{gathered}
$$

and for

$$
\begin{gathered}
z=|z| \mathrm{e}^{\mathrm{j}\{\theta\}}, \\
F(z)=\operatorname{LOG}|z|+\{\theta\} \mathrm{rad} \\
=\ln |z| \mathrm{Np}+\{\theta\} \mathrm{jNp} \\
=(\ln |z|+\mathrm{j}\{\theta\}) \mathrm{Np} .
\end{gathered}
$$

In electrotechnology, if the input of a two-port network has the complex amplitude $S_{1}$, and its output, $S_{2}$, then the logarithm of the ratio $S_{1} / S_{2}$ is called the "transfer exponent" of the network. (For a transmission line, the transfer exponent per unit length is called the "propagation coefficient.") Publication 27-2 of the International Electrotechnical Commission expresses the transfer exponent as $\Gamma=A+j B$, with a footnote on the unit of $\Gamma$ that, in general, units are used only with $A$ and $B$ separately. Thus $A$ is expressed in nepers, and $B$ in radians, and $\Gamma$ involves a mixture of units. Equations (38) and (43) show that $\Gamma$ can be considered to be dimensionally homogeneous, being expressed as a complex number times a radian or a neper. 
Formulas (42) for $f(\theta)$ and $f(L)$ involve the explicit appearance of units of $\theta$ and $l$, a feature that is undesirable in quantity relations. This situation arises from the original stipulation that $f(X)$ be numerical for class $\mathrm{C}$ functions, and is associated with the definition

$$
\mathrm{e}^{x}=\exp x \stackrel{\mathrm{d}}{=} \sum_{0}^{\infty} x^{n} / n !
$$

and the Euler formula

$$
\mathrm{e}^{\mathrm{j} x}=\cos x+\mathrm{j} \sin x
$$

for numerical $x$.

Equation (45) can be generalized [3] to the quantity equation

$$
\mathrm{e}^{\mathrm{j} \theta}=\cos \theta+\mathrm{j} \sin \theta
$$

if we assign the dimensional properties

$$
\begin{gathered}
D^{2}(\theta)=D(1) \\
D(\sin \theta)=D(\theta) \\
D(\cos \theta)=D(1)
\end{gathered}
$$

The function $\mathrm{e}^{\mathrm{j} \theta}$ becomes a dimensional hybrid, i.e., it is a vector having components in two dimension spaces. This mixed dimensionality is, however, admissible in (18a), since (47) implies that elements of the form $a+\mathrm{j} b \theta$ comprise a multiplicative group.

Analogously,

$$
e^{L}=\cosh L+\sinh L
$$

with

$$
\begin{gathered}
D^{2}(L)=D^{2}(\theta)=D(1) \\
D(\sinh L)=D(L) \\
D(\cosh L)=D(1)
\end{gathered}
$$

In general, the exponential function of a nonnumeric quantity provides an artifice for the simultaneous treatment of differentially related quantities of different dimensions. Those hybrid quantities comprise a hypercomplex algebra on a subspace of the vector space of dimensions; they are elements of a division ring, and isomorphic to $2 \times 2$ matrices.

\section{Conclusions}

Angle and logarithm are interrelated dimensionally, as well as analytically. When radian is considered a base unit, neper becomes a derived unit, and should be considered the SI unit for logarithmic quantities.

\section{References}

[1] Page, C. H., Physical Entities and Mathematical Representation, J. Res. Nat. Bur. Stand. (U.S.), 65B (Math. Sci.), No. 4, 227-235 (Oct.-Dec. 1961).

[2] The International System of Units, Nat. Bur. Stand. (U.S.) Spec. Publ. 330, p. 2 (1974 ed.).

[3] Page, C. H., The Mathematical Representation of Physical Entities, IEEE Trans. Educ., E-10, 70-74, June 1967.

(Paper 79B3\&4-428) 